\title{
Flood warning systems and ubiquitous computing
}

\author{
Philippe GOURBESVILLE ${ }^{*}$, Jelena BATICA ${ }^{1}$ \\ Jean Yves TIGLI ${ }^{2}$, Stephane LAVIROTTE², Gaetan REY² \\ Durairaju Kumaran RAJU ${ }^{3}$
}

\author{
1. University Nice Sophia Antipolis / Polytech Nice Sophia / URE Innovative CiTy, 930, route des Colles - 06930 Sophia Antipolis - France \\ philippe.gourbesville@unice.fr \\ 2. I3S - CNRS \& University Nice Sophia Antipolis - France \\ 3. TMSI - National University Singapore - Singapore
}

\begin{abstract}
Floods in Asia represent the first natural hazard. During the last five years, major events have affected more than 600 millions in Asian countries and constitute one of the main obstacles to the economic and social developments. In order to address part of the flooding challenge, the use of ICT can be promoted according to the type of flooding and local situation of IT environment. In a very classical approach like the SCADA systems, most of the decision making process takes place after a centralization of data and some simulations produced by one or several models. In such case, the reaction time requests at least several tens of minutes which are already longer than the flooding process that may append in less than 10 minutes after the start of the rain. The UbiFLOOD project deals with implementation of ubiquitous computing in flood warning and forecasting systems in different Asian background and the aim of the UbiFLOOD project is to implement ubiquitous solutions in the existing flood warning and forecasting systems. As an outcome, the framework will be developed with the main purpose to alert the population regarding flood alert. Since ubiquitous computing in flood warning systems is not widely applied in Asia, the new proposed solution within the framework should result in better operational organization and management of warning systems.
\end{abstract}

Key words : Flood warning systems, flood forecasting, SCADA, ICT, ubiquitous computing, sensors, distributed approach.

\section{Systemes d'annonce de crue et informatique ubiquitaire}

RÉSUMÉ. - En Asie, les inondations représentent les premières des catastrophes naturelles. Au cours des cinq dernières annèes, les évènements majeurs ont affectésplus de 600 millions de personnes dans les pays asiatiques et constituent l'un des principaux obstacles l'évolution économique et sociale. Afin de répondre à une partie du défi des inondations, l'utilisation des Technologies de l'Information et de la Communication et en particulier celles relatives à l'approche ubiquitaire peut être promue en fonction du type d'inondation et de la situation de l'équipement informatique dédié à l'annonce de crue. Dans une approche classique comme dans les systêmes SCADA, la plupart des processus de décision interviennent après une centralisation des données et des simulations produites par un ou plusieurs modéles. Dans ce cas, les temps de réaction demandent au moins plusieurs dizaines de minutes et sont donc bien supérieures à la dynamique des écoulement qui peuvent se développer en moins de 10 minutes après le début des précipitations.

Le projet UbiFLOOD repose sur la mise en œuvre de l'informatique ubiquitaire pour l'alerte et la prévision des inondations dans plusieurs environnements urbains asiatiques. Le but de ce projet est de mettre en ouvre des solutions ubiquitaires pour les systèmes d'alerte et de prévisions. Le système, basé sur la nouvelle génération de capteurs communiquants et ubiquitaires, a pour objectif principal la mise en place d'une communication rapide à destination des personnes exposées au risque inondation. L'approche ubiquitaire est encore peu développée dans les systèmes d'annonce de crue mais représente potentiellement une voie d'amélioration notable des dispositifs classiques et devrait logiquement permettre une meilleure organisation opérationnelle ainsi qu'une gestion efficace des systèmes d'alerte.

Mots-clefs : Système d'annonce de crue, prévision d'inondation, SCADA, TIC, capteurs informatiques ubiquitaires, approche distribuée

\section{BACKGROUND}

Floods in Asia represent the first natural hazard. During the last 5 years, major events have affected more than 600 million inhabitants in Asian countries and constitute one of the main obstacles to the economical and social developments

\footnotetext{
* Corresponding author
}

The recent events of Pakistan and in Thailand have demonstrated the magnitude of the hydrological processes and the consequences for the impacted communities. Many factors contribute to make Asia the most flood prone area of the world: size of the catchments and rivers, extreme rainfall events, growing urbanization, changes in land use, etc.

In order to address part of the flooding challenge, the use of Information and Communication Technologies (ICT) can be promoted according to the type of flooding and local 
situation of IT environment. Many already existing examples may be provided to illustrate this major trend which is closely supporting the growing position of hydroinformatics approaches and methods [Gourbesville, 2009; Gourbesville 2011]. The need for efficient warning systems related to flood is now clearly expressed especially in the growing complexity of urban environments like those of the megacities. The combined development of new generations of sensors and new communication protocols allows revisiting the concepts used until now in the flood warning disposals. The efficiency of these systems has been serious questioned during recent events and improvements are serious expected in order to provide an real support to the exposed populations.

Most of the flood warning systems already implemented are based on the concepts used in the Supervisory Control and Data Acquisition (SCADA) systems. Originally, these systems have been designed and implemented for monitoring and controlling a plant or industrial equipment such as telecommunications, water and waste water control, energy production, oil and gas refining and transport... A SCADA system (Figure 1) usually consists of several subsystems which are:

- a Human Machine Interface (HMI) is the apparatus which presents process data to a human operator, and through this, the human operator monitors and controls the process;

- a supervisory system based, acquiring and gathering data on the process and sending commands to the process;

- several Remote Terminal Units (RTUs) connecting to sensors in the process, converting sensor signals to digital data and sending digital data to the supervisory system;

- several Programmable Logic Controller (PLCs) used as field devices because they are more economical, versatile, flexible, and configurable than special-purpose RTUs;

- a communication infrastructure connecting the supervisory system to the remote terminal units;

- and various process and analytical instrumentation.
SCADA systems are clearly data acquisition oriented and they could be associated to Distributed Control Systems (DCS) that are process oriented and driven. SCADA refers to centralized systems which monitor and control entire sites, or complexes of systems spread out over large are (anything from an industrial plant to a nation). Most control actions are performed automatically by RTUs or by PLCs. Host control functions are usually restricted to basic overriding or supervisory level intervention. For example, a PLC may control the flow of cooling water through part of an industrial process, but the SCADA system may allow operators to change the set points for the flow, and enable alarm conditions, such as loss of flow and high temperature, to be displayed and recorded. The feedback control loop passes through the RTU or PLC, while the SCADA system monitors the overall performance of the loop.

In the flood warning systems, the SCADA are frequently associated with hydrological/hydraulic modeling tools which represent the support to the decision and the link to the DCS. However, this strategy considers that the concepts implemented in SCADAs may operate in the specific domain of flood warning. If the general principles may be accepted without difficulty, the specificity of some flood events - extremely short concentration time (below 10 minutes) and fast propagation in urban environments request an immediate answer and information of the public which is exposed to the flooding process. In such situations, obviously, the centralized approach with supervision and monitoring can't match the expectations and provide an efficient answer to the exposed population. The time for analysis, mainly based on human expert analysis, request a duration which is not compatible with the speed of the process. Many examples can be provided to illustrate such situation like the flood events recorded in November 2011 in Genoa, Italy.

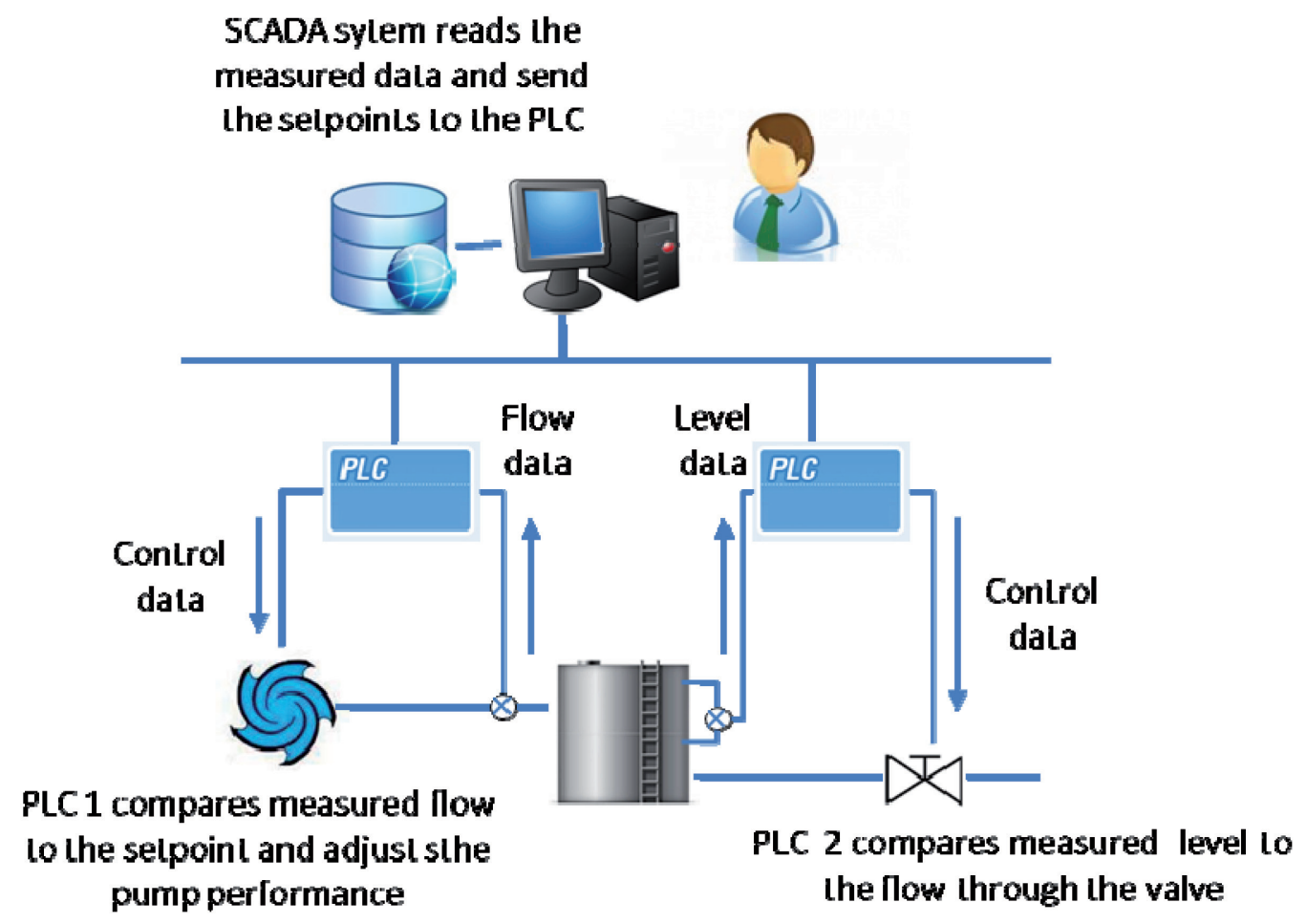

Figure 1: Principle of SCADA applied to a water system. 


\section{FROM CENTRALIZED TO DISTRIBUTED \& UBIQUITOUS ARCHITECTURE}

In order to improve performance of flood warning systems especially in the case of fast processes like urban flash floods, a shift of paradigm is needed for providing instantaneous information to the exposed populations and with the right message. The development of new generations of sensors allows monitoring urban streams with a high level of accuracy and for a limited cost. These new sensors have now communicating capacities which are embedded in a similar way to all modern communicating devices. The development of such technology allows introducing the concept of Ubiquitous Computing which represents an alternative to the centralized approach actually widely used.

Ubiquitous Computing refers to omnipresent computers that serve people in their everyday lives at home, at work, in transports... Ubiquitous Computing was introduced by Mark Weiser in 1991, raised many challenges across computer science: in systems design and engineering, in systems modeling and in user interface design. But, initially the effective integration and interaction with the physical world sufficed to promote Ubiquitous Computing interest due to significantly increased real world visibility as well as real world control, towards ambient intelligence. These first ubiquitous systems were often proofs of concept with a single static configuration with a priori known devices. Ubiquitous Computing is now become a reality for applications evolving in the real environment thanks to the evolution of ICT technologies: consumer electronics, telecommunications and software.

With the new devices and their functionalities, Ubiquitous Computing can now revolutionize interactions between users, their environment and information systems. It offers a post-desktop model for human/machine interfaces to access to information systems not only through laptop, PDA and mobile phone, but also through each equipment used in the daily life: alarms within buildings, displays networks in cities, etc. As devices become smaller, more connected and more integrated into a global environment which is developing continuously, the technology disappears into our surroundings in everyday life objects and devices. Thus ubiquitous systems can open up new areas of applications: smart buildings, industrial automation, healthcare, power distribution and host of others... This interconnection of devices and objects is expected to amplify the profound effects that networked systems are having on our modern society. The emergence of intelligent cities is one of the main societal impacts of Ubiquitous Computing. Indeed, the city now has the technology to improve public safety through a better management of natural risks. Especially, the efficiency of warnings to the population can be improve tremendously, by increasing the number of informed people, by improving the relevance of broadcast messages, by reducing the time required for communication with the public. With networked devices in the smart cities, warning systems can become, opportunistically, richer than ever, mainly in data collection from the field and for communications with the public.

\section{THE UBIFLOOD APPROACH}

In order to explore the new possibilities offered by the ubiquitous approach, a research project called UbiFLOOD [http://www.ubiflood.eu/] has been launched with the objectives to develop some ubiquitous approach in different Asian cities which are exposed to regular flooding events. Case study areas within the project are Bangkok, Mumbai and Singapore. The common characteristics for selected cities are that they are in monsoon region and that they have a history of flooding problems. However characteristic of flood events are very different (from slow to extremely fast) and require specific answers.

Following the analysis made in Mumbai and in Singapore, it appears that one of the most severe difficulties is generated by flash floods events that may appear in less than 10 minutes after the start of intense rainfall events. Singapore authorities are deploying a complete data acquisition chain including radar data treatment in real time but, despite this action, the city center has been exposed to flooding in 2010 and 2011 [Recorded videos are available on the UbiFLOOD website at: http://www.ubiflood.eu/].

In order to cope with such situations, a potential solution is to deploy a set of sensors - autonomous and low cost able to collect information about water levels and to communicate directly to the exposed population by SMS, email and voice messages. This approach allows to react faster and to avoid the delay due to the centralized concept of the traditional warning system. The principle of the new systems is presented on the figure 2 .

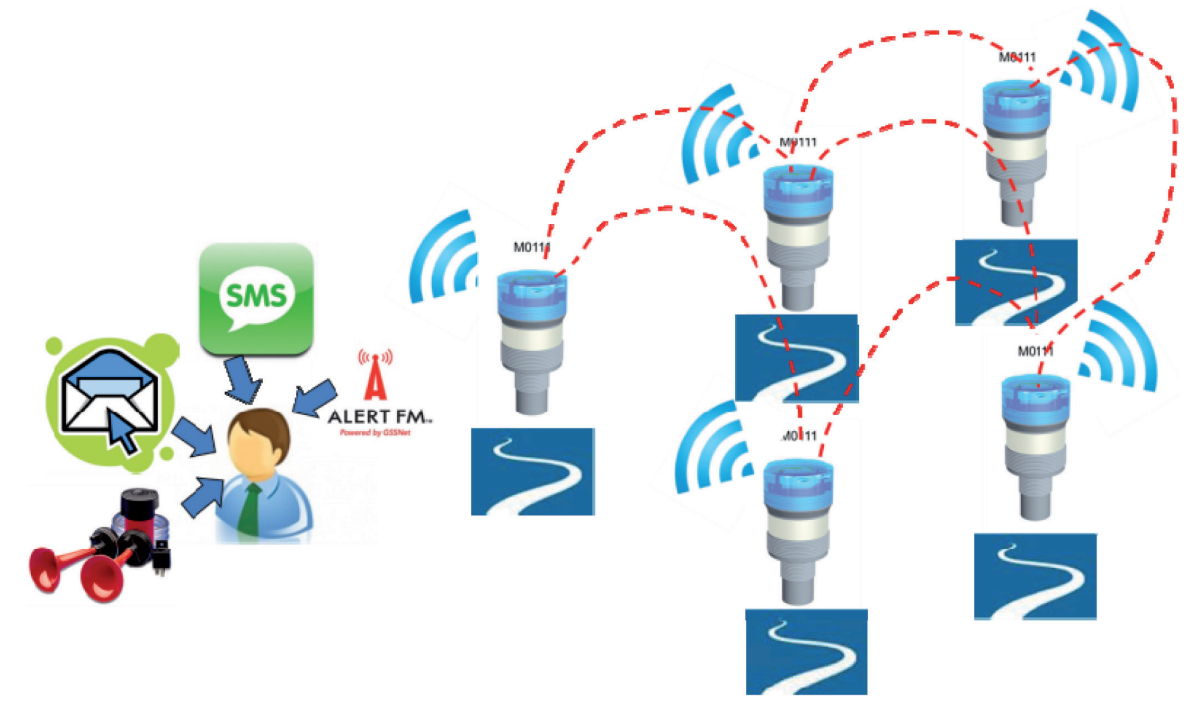

Figure 2: Architecture of the UbiFLOOD solution based on Ijinus sensors. 


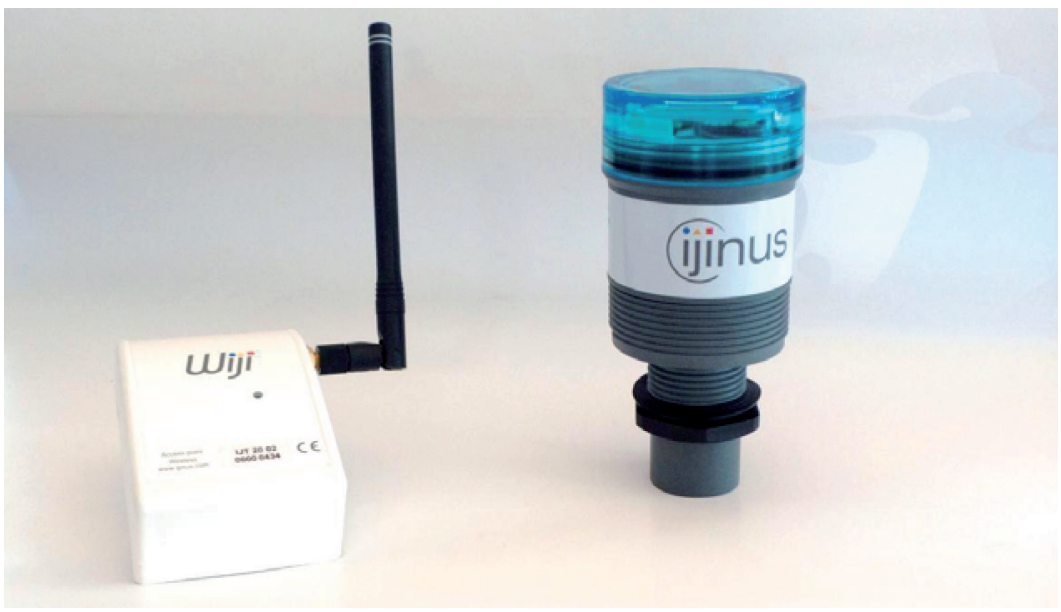

Figure 3: Ijinus ultrasonic sensor used as validation prototype.

In order to validate the concept, a prototype based on Ijinus communicating sensors [http://www.ijinus.com] wireless ultrasonic numerical level probe - has been developed and tested in laboratory, in Singapore. The ultrasonic sensors used $-120 \mathrm{KHz} 0.2$ to $3 \mathrm{~m}$ - are providing an accurate measure on the water level with minimum resolution of $2 \mathrm{~mm}$. The autonomy of the sensor can reach up to one year with the internal battery. With these characteristics, the installation and the maintenance of the sensors represents a limited investment which allows redundancy in order to optimize the functionalities of the network. The sensors are connected to the communication network and can directly send information to the surrounding devices according to the defined protocol.

In order to validate the concept, a prototype based on Ijinus communicating sensors [http://www.ijinus.com] wireless ultrasonic numerical level probe - has been developed and tested in laboratory, in Singapore. Some videos of the conducted experiments are presented on the UbiFLOOD website [http://www.ubiflood.eu/] and describe how the data acquisition process is able to send a alert message to a mobile phone according to the defined rules.

\section{TECHNOLOGICAL SOLUTION: SERVICE ORIENTED \& SELF-ADAPTED SOFTWARE SYSTEM}

Service-Oriented Architecture [Durairaju Kumaran et al., 2010] [Mike et al., 2007] (SOA) is a well-known set of principles and methodologies for designing and developing software in the form of interoperable services. SOA defines how to integrate widely disparate applications for a Web-based environment, over Internet and uses multiple implementation platforms. Rather than defining an API, SOA defines the interface in terms of protocols and functionality. Service orientation requires loose coupling of services with operating systems, and other technologies that underlie applications.

Under pressure from the ubiquitous computing, many standards in the business are appearing to promote new concepts like Machine to Machine [Lawton, 2004] (M2M), Internet of Things [Mattern F., Floerkemeier C., 2010] (IoT), and more recently the Web of Things (WoT), to federate service-oriented approaches and software development using networked physical devices. The used approach is based on these recent advances. These standards and/or well-known technologies are used to implement Services for Devices oriented architectures. For example UPnP (Universal Plug and Play from UPnP consortium [UPnP consortium]) and DPWS (Device for Web Services from OASIS [DPWS, 2009]) allow devices to connect easily and simplify the implementation of networks in the restricted area like smart buildings (file sharing, communications, and entertainment) or in companies. It allows defining and publishing UPnP and DPWS control protocols on top of standard communication of the Internet.

Both uses XML based protocols above Web protocols to manage services provided by networked physical devices. These protocols allow two kinds of communications between services of devices and software clients: classical remote methods invocations but also events based communications much more adapted to device that must be able to collect spontaneous information in the physical environment. Moreover they introduce protocol to manage appearance, discovery and disappearance of services attached to device, at runtime. Every device is then usable without knowing before what the services it provides are and their interface. Thus, ubiquitous applications can be deploy on partly unknown set of devices that can be change at runtime.

The implemented solution is founded on an original approach based on services for devices software infrastructure to self-adapt at runtime any ubiquitous application to the change and the availability of surrounding devices [Durairaju Kumaran et al., 2010] [Tigliet al., 2009]. This approach is based on a set of independent rules to describe how the system must react and evolve when the software infrastructure is changing. Then main theoretical challenges address the interferences and even conflicts that may appear between rules based modifications on the shared software application. Indeed the merge algorithm between the modifications to finally modify the common application must maintain the its consistency, i.e. verify proved properties during merging process like mastered response time for the adaptation, some logical and semantic properties described as requirements for the consistency of the application.

In the case of flood warning and management system, software infrastructure consist in networked sensors, simple pre-computed rules to define in real time in which geographical area warning messages have to be sent to the population. In the scenario, the sensors provide real time water level data which are more reliable than forecasted data. The flooding area / zone is calculated from the pre-created 


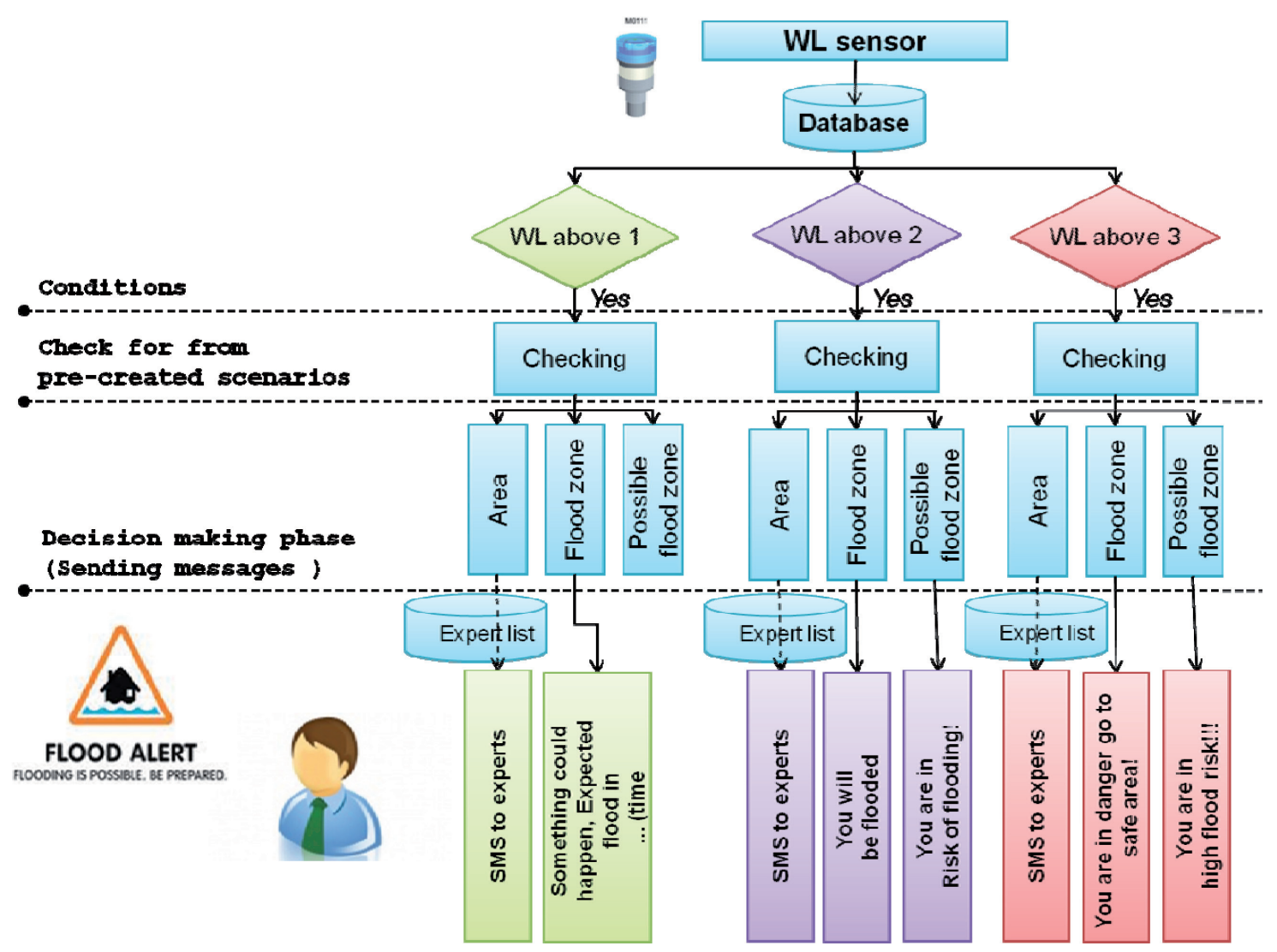

Figure 4: Example of communication protocol and rules implemented into the Ijinus sensor.

scenario. In the validation case developed in Singapore, two kinds of services and devices are used to send information about flooding. The first set is dedicated to expert users in order to help them to analyze and assess the situation. The second set is composed with various communication devices and services is dedicated to send warning to the public.

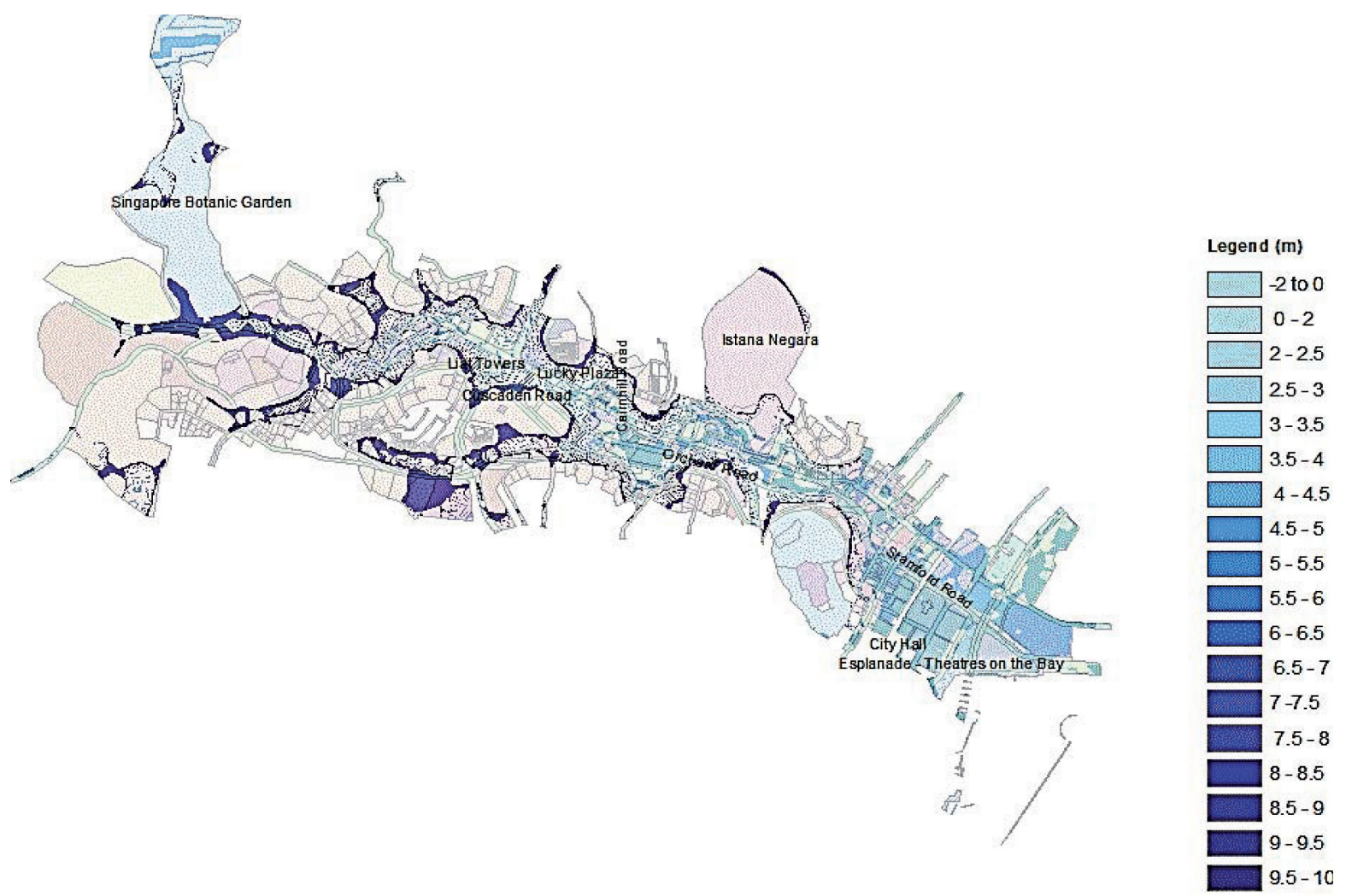

Figure 5: Example of flood map in Singapore sent to the public on smartphones. 
Population can be informed through a web page, aSMS/ MMS and/or an Email. The map displayed on the web page can be modified with real time information on the water levels and discharges collected from the monitoring network. System send SMS/MMS alert on the following cases:

1. SMS / MMS alert will be sent to all government officials / experts in the flooded admin zone based on their phone model. The information to those officials / experts will be stored in the database.

2. SMS / MMS alert will be sent to registered public. Public can register to get alert on their zone of interest. While registering, they need to provide phone model. This alert is not on location based. For example, if he /she registers for zone A he/she will get information only for zone A even though he/she may not be there while flood in that zone.

3. SMS / MMS alert to registered users. He/she doesn't want to register for a particular zone. This alert is location based service. That is, if he/she registers with the system, he/she will get flood alert if he /she is in the flooded zone.

The set of rules to self-adapt the ubiquitous application for the flood warning are then:

1. To send a SMS according to the alert level;

2. To send an email according to the alert level;

3. To modify the map on the web page with the water level;

4. To visualize the evolution curve of the water level at a specific location;

5. To visualize the evolution of the water level on a $2 \mathrm{D}$ map; 6 . To visualize the location and the status of the sensors on Google map.

Of course each of these rules are applied when required devices and services are available.

\section{CONCLUSION \& PERSPECTIVES}

The ubiquitous approach supported by new sensors and interfaced with communicating devices allows potentially reshaping the flood warning systems and could provide an efficient answer for flood events such like in Singapore where the concentration time doesn't exceed 10 minutes. The communicating capacity of the individual devices allows communicating immediately to the exposed population and to inform about on-going risks. However, this progress needs to be associated to a very careful definition of the messages to send to the population in order to generate the proper reaction. At the same time, the data collection has to be carried out in order to nourish a knowledge base which is the key reference for establishing the rules for issuing warning messages. In such context, the future of warning system has to be seen as a combination of the traditional SCADA approach combined with the ubiquitous devices which represent a great potential of improvement. The availability of both sensors and communication devices like smartphones allow to review in depth the concept of warning system with the associayed workflows.

The upcoming developments of the UbiFLOOD project will allow to explore further these tracks and to evaluate the practical implementation of such solution in Asian cities. The next step for the project is to implement in each sensor the possibility to run a simplified hydraulic model able to identified the closest flood map to the on-going situation and to send an alert message according to the situation to the exposed persons. This last step will strongly improve the fficiency of the disposal and will allow to reach a better awaremess and preparedness of the population.

The key question of the mobile devices - mainly mobile phones - used by the population and their heterogeneity will remain and represent a large domain for investigation and experiments. This issue is planned to be addressed in next phases on the UbiFLOOD development.

\section{ACKNOWLEDGMENTS}

The Ubiflood project [http://www.ubiflood.eu/] is funded under the STIC Asie framework which is designed to encourage collaborative research and academic exchanges between France and countries from Asia.

\section{REFERENCES}

Gourbesville P. (2009) - Data \& hydroinformatics: new possibilities and new challenges. Journal of Hydroinformatics. 11 (3-4) : 330-343

Gourbesville P. (2011) - ICT for water efficiency, Environmental Monitoring, Intech. 411-426

Durairaju Kumaran R., Clews E., Tigli J.Y., Lavirotte S., REY G. (2010) - Sensors Networks, SOA and Web Based Approach for Fresh Water Environmental Monitoring. 9th International Conference on Hydroinformatics (HIC), Tianjin, China, 7-11 September 2010.

Ferry N., Lavirotte S., Tigli J.Y., Rey G., Riveill M. (2010) Toward a Behavioral Decomposition for Context-awareness and Continuity of Services. Proceedings of the International Symposium on Ambient Intelligence (ISAmI), Springer, Guimarães, Portugal. 55-62

Tigli J.Y, Lavirotte S., Rey G., Hourdin V., Cheung D., Callegari E., Riveill M. — WComp middleware for ubiquitous computing: Aspects and composite event-based Web services. Annals of Telecommunications, ed Springer Paris. 64(3-4) : 1958-9395

Mike P. Papazoglou M.P., Heuvel W.J. (2007) — Service oriented architectures: approaches, technologies and research issues. The VLDB Journal. 16 : 389-415

Lawton G. - Machine-to-Machine Technology Gears Up for Growth. Computer. 37(9) : 12-15

MAtTern F., Floerkemeier C. - From the internet of computers to the internet of things. From active data management to event-based systems and more, Kai Sachs, Ilia Petrov, and Pablo Guerrero (Eds.). Springer-Verlag, Berlin, Heidelberg.

DPWS Specification OASIS: http://docs.oasis-open.org/ws-dd/ns/ dpws $/ 2009 / 01$

UPnP consortium: www.upnp.org 